Tipología y usos del suelo en agroecosistemas del valle del guamuez, Putumayo - Colombia

Juan Antonio Espinosa-Alzate, Tomás Enrique León-Sicard, Leonardo Alberto Ríos-Osorio

\title{
TIPOLOGÍA Y USOS DEL SUELO EN AGROECOSISTEMAS DEL VALLE DEL GUAMUEZ, PUTUMAYO - COLOMBIA
}

\author{
Typology and land use in agroecosystems of Guamuez Valley, Putumayo, Colombia
}

\author{
Juan Antonio Espinosa-Alzate \\ Universidad de Antioquia, Medellín, Antioquia, Colômbia \\ jespinosaalzate@gmail.com \\ Tomás Enrique León-Sicard \\ Universidad Nacional de Colombia, Bogotá, Colombia \\ teleons@unal.edu.co \\ Leonardo Alberto Ríos-Osorio \\ Universidad de Antioquia, Medellín, Antioquia, Colômbia \\ 1rios_01@hotmail.com
}

Artigo recebido em 27/02/2014 e aceito para publicação em 07/01/2015

RESUMEN: Esta investigación tuvo como objetivo analizar diferentes agroecosistemas existentes en una región del departamento de Putumayo (Colombia) y su relación sociocultural con los contextos productivos y de conservación del territorio, con base en los elementos conceptuales y metodológicos que ofrece la agroecología. Para ello se diseñó un estudio de métodos mixtos por el cual se aplicaron metodologías de análisis de suelos y valoración de aspectos culturales por medio de herramientas cualitativas tipo entrevista y grupos de discusión. En el análisis se tuvo como base el concepto de estructura ecológica principal del paisaje (EEP) y principios de la agroecología, orientado por un análisis etno-agrícola del fenómeno. Como resultado se identificaron tres tipos de sistemas productivos, donde coexisten grupos de indígenas con colonos y población flotante que han desarrollado diferentes procesos de adaptación ecosistémicos. Esta heterogeneidad implica, por lo menos, dos modos de vida diferentes: uno, de tipo "endógeno", campesino - colonos y ligado con las comunidades indígenas, y el otro, mediado por la visión clásica del desarrollo económico. En este sentido, todos los habitantes, sin importar en qué modo de vida se inscriben, se ven abocados a superar las limitantes biofísicas y ecosistémicas del territorio, agravadas en la región por los procesos de tala y mal uso de las tierras, que han generado, en muchos sectores, procesos severos de degradación del medio natural incluyendo fenómenos irreversibles de erosión en surcos y cárcavas. Este deterioro se expresa en disminuciones que limitan significativamente las posibilidades de subsistencia y de acumulación de los habitantes locales.

Palabras Clave: Agroecología. Cultura. Putumayo. Territorio. Usos del Suelo.

ABSTRACT: The objective of this research was to analyze different agroecosystems located in a region of the Departamento de Putumayo (Colombia) and its sociocultural relationship with productive and land conservation contexts, based on the conceptual and methodological aspects of agroecology. A mixed methods study was carried out, and soil testing methodologies and assessment of cultural aspects with 
research tools such qualitative interview and focus groups, were designed. The analysis was based on the concept of principal ecological landscape structure (PELS) and agroecology principles, guided by an ethno - agricultural analysis of the phenomenon. Three types of production systems, where indigenous groups coexist with settlers and migrant population who have developed different processes of ecosystem adaptation, were identified. This heterogeneity implies at least two different livelihoods: first, "endogenous type" in peasant - settlers linked to indigenous communities, and second, mediated by the classical view of economic development. All people, no matter what livelihoods have, are forced to overcome biophysical and ecosystem limiting of territory, indeed the processes of forest logging and poor land use, which have generated severe processes of environmental degradation including irreversible phenomena of rill and gully erosion. This deterioration is expressed in limiting subsistence possibilities and accumulation decrease of local inhabitants.

Keywords: Agroecology. Culture. Land Use. Putumayo. Territory.

\section{INTRODUCCIÓN}

Colombia es un país de una gran variedad ecosistémica, geográfica y cultural que no es ajeno a la problemática mundial de la degradación de tierras. Su uso y aprovechamiento ha generado fuertes procesos de degradación de suelos por distintos fenómenos como erosión, desertificación, salinización, compactación y decrecimiento de la fertilidad.

Según la FAO (1995), la degradación de la tierra es la reducción en su capacidad para proporcionar bienes y servicios ecosistémicos y garantizar sus funciones durante un determinado período de tiempo para sus beneficiarios. En estricto rigor, este término es más amplio que el de erosión o desertificación y denota pérdida de la capacidad productiva de la tierra.

De otra parte, la degradación de tierras puede entenderse como la acción de un conjunto de factores tanto de índole biofísico como antrópico, que desencadenan alteraciones de las cualidades y características de la tierra, incluyendo al conjunto de suelos, coberturas vegetales, fauna asociada y dotaciones de agua que existen dentro de determinados paisajes fisiográficos y que afectan la calidad de vida de las poblaciones urbanas y rurales. (LEÓN, 2002)

La agroecología se define de forma tradicional como la ciencia que provee los conceptos y principios ecológicos y culturales para el diseño y manejo de agroecosistemas sostenibles; en este abordaje de la agroecología, la cultura, como concepto orientador, adquiere la connotación de proceso adaptativo y transformador de los seres humanos sobre los ecosistemas, en términos de estructura simbólica, organización social, económica y política y de plataforma tecnológica (ANGEL, 1993; 1995; 1996)

La idea de la agroecología es ir más allá del uso de prácticas alternativas y desarrollar agroecosistemas con una dependencia mínima de energía y de insumos externos (agroquímicos y fertilizantes), enfatizando en sistemas agrícolas complejos en los cuales las interacciones y sinergismos ecológicos y culturales entre sus distintos componentes proporcionen, entre otras cosas, los mecanismos para que los sistemas incorporen su propia fertilidad de suelo, productividad y protección del cultivo. (ALTIERI, 1995).

Además de suministrar una base científica para alcanzar una productividad sostenible, la agroecología enfatiza en la capacidad de las comunidades locales para innovar, evaluar, y adaptarse a condiciones heterogéneas extremas, a través de métodos de investigación participativa y de extensión campesino a campesino. Las tecnologías agroecológicas se enfocan sobre la diversidad biológica y cultural, la sinergia, el reciclaje e integración y sobre los procesos sociales que valoren la participación de la comunidad, que es clave, pues el desarrollo del recurso humano es la piedra angular de cualquier estrategia que apunte a aumentar las opciones de la gente rural y sobre todo de agricultores de escasos recursos (GLIESSMAN, 1998).

En la región del piedemonte del Putumayo, zona sur de Colombia, coexisten comunidades indígenas con colonos y población flotante que tienen modos de vida diferentes y los cuales reflejan dichas diferencias en las formas en las que ocupan y usan el suelo, lo cual ha llevado históricamente a la 
presentación de distintos fenómenos de degradación del mismo.

El objetivo de esta investigación fue describir las características de algunos agroecosistemas específicos del piedemonte amazónico colombiano a partir de análisis culturales que incluyen las lógicas sociales de apropiación del territorio de las comunidades campesinas.

\section{MATERIALES Y MÉTODOS}

\section{Tipo de Estudio}

Se diseñó un estudio de métodos mixtos con la aplicación de herramientas de evaluación del suelo y métodos de análisis cualitativos tipo entrevista y grupo de discusión, con los cuales se obtuvo información sobre el manejo y diseño de los agroecosistemas.

\section{Area de Estudio}

El área de estudio se encuentra localizada en los municipios de Puerto Asís, La Hormiga y Orito entre las coordenadas geográficas 535.000, $560.000 \mathrm{mN}$ y $1.010 .000,1.064 .000 \mathrm{mE}$ ocupan una superficie de $5.348 \mathrm{~km}^{2}$, su altitud media es de 292 msnm. El recorrido realizado en la zona correspondió a $82.5 \mathrm{~km}$ (ver Figura 1).

FIGURA 1: Recorrido en la zona del Putumayo por los municipios de Puerto Asís, La Hormiga y Orito, ubicados en la región del Valle del Guamuez

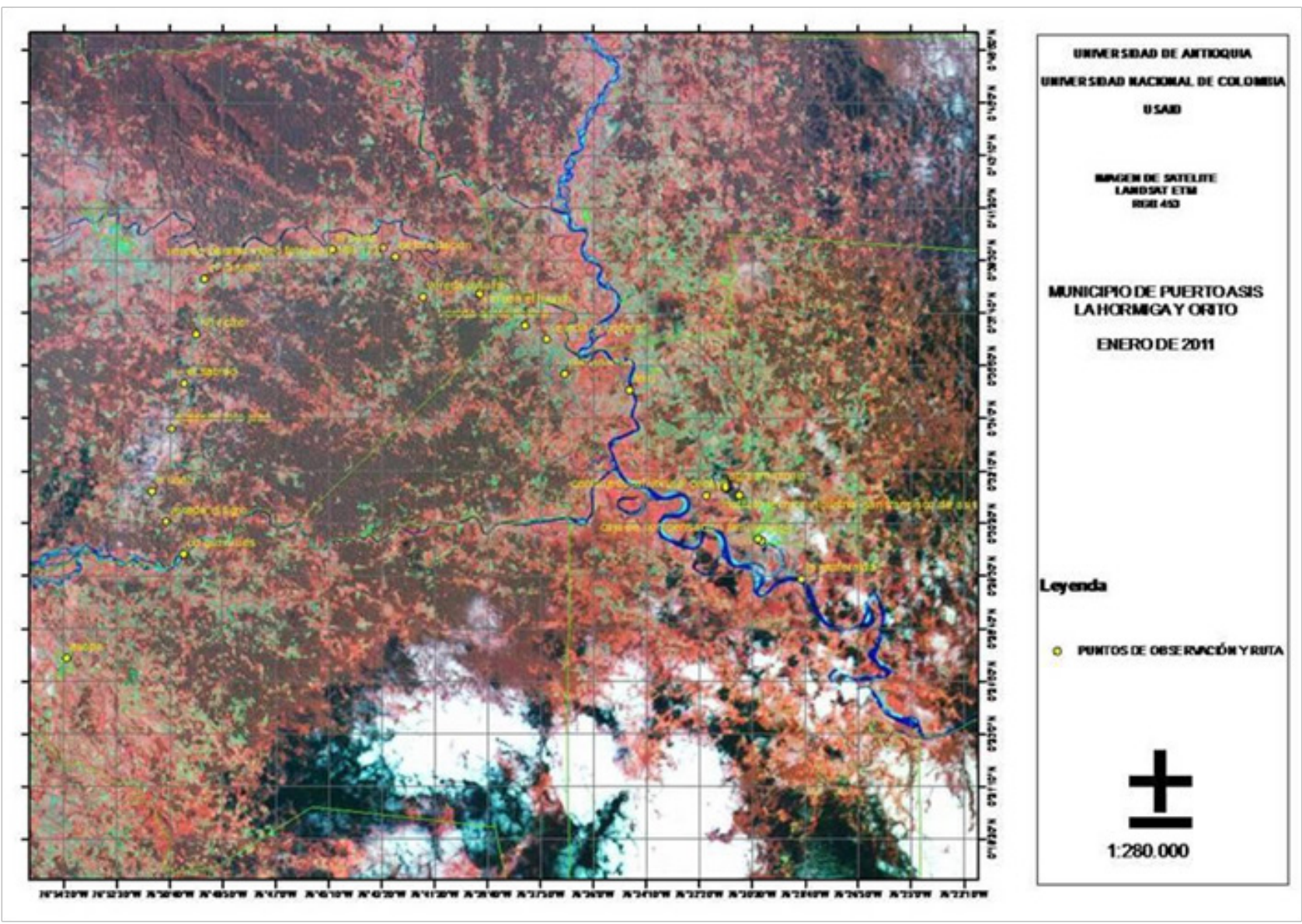

Fonte: Satélite Landsat ETM, 2013. Organização dos autores 


\section{Sistemas productivos}

Para la descripción de los procesos ecosistémicos productivos y culturales se realizaron procedimientos convencionales de cartografía utilizando sistemas de posicionamiento geográfico. La descripción de los tipos de suelos se realizó a partir de observaciones de perfiles en taludes o en

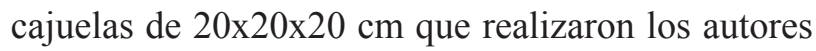
en los recorridos por la zona de estudio.

Se utilizó el concepto de estructura ecológica principal (EEP) propuesta por Van Der Hammen y Andrade (2003), definida como: “... la red de espacios y corredores que sostienen y conducen la biodiversidad y los procesos ecológicos esenciales a través del territorio, en sus diferentes formas e intensidades de ocupación, dotando al mismo de servicios ambientales para su desarrollo sostenible...". De igual forma se realizó un análisis desde la agroecología, sobre el conocimiento local relacionado con los usos del suelo, que ha sido un elemento importante para reevaluar los supuestos de los modelos coloniales y agrícolas de desarrollo.

\section{Análisis Sociocultural de los Usos del Suelo}

Se realizaron entrevistas en profundidad y grupos de discusión definidos a partir de categorías conceptuales derivadas de los principios de la agroecología, teniendo en cuenta el establecimiento de relaciones de interdependencia entre los diferentes factores formadores del paisaje y los elementos que conforman los sistemas de producción para poder obtener un mejor conocimiento del manejo de los ecosistemas de la región por parte de cada grupo humano.

En el proceso de recolección de información se realizaron 3 visitas de campo a diferentes sistemas productivos de pequeños agricultores en el Valle del Guamuez y se desarrollaron 3 entrevistas semiestructuradas, teniendo como guía las categorías conceptuales: territorio, producción, conservación, biodiversidad, cultura, seguridad alimentaria y comercialización.
La tipología de productores incluidos en el estudio fue la siguiente: 1), Pequeño productor ganadero de Pto Asis, en zonas planas y en reconversión hacia sistemas silvopastoriles con introducción de banco de proteínas para forraje. 2) Pequeños productores de pimienta en la vía que conduce al Valle del Guamuez, beneficiarios del proyecto ADAM- USAID, quienes son productores colonos mestizos y afrodescendientes que trabajan sistemas productivos de economía campesina diversificados, en asocios Pimienta - maíz - yuca, frutales-maíz - forestales. Y 3) Productores del municipio de la hormiga que trabajan en sistemas de producción de cacao y sistemas diversificados entre silvopastoril y cacao-platano-frutales.

Con base en las categorías conceptuales planteadas se realizaron 2 grupos de discusión con productores beneficiarios de proyectos de cooperación internacional y con representantes de instituciones públicas, privadas y organizaciones no gubernamentales relacionadas con el sector agrícola de la región, entre ellas: CORPOAMAZONIA (autoridad ambiental de la región), Condimentos Putumayo, AGROAMAZONIA (asociación de productores), ASOPA (Asociación de Productores de Cacao), Comité de Ganaderos de Putumayo, ADAM (desarrollo alternativo municipal), COOFORTALEZA (cooperativa de productores), ASOPEZ (asociación de productores piscícolas), ASOMAYO (asociación de productores del Sur de Putumayo), CORPOSUR (asociación de productores) y ASOYAIP (asociación de productores).

\section{RESULTADOS}

\section{Descripción de la zona de estudio}

La zona se caracteriza por un relieve de lomerío con diferentes grados de disección y valles aluviales de los ríos Orito y Guamuez que desembocan en el río Putumayo. Al sur del río Guamuez se presentan suelos Inceptisoles y al norte suelos Oxisoles o Ultisoles.

El área presenta precipitación promedio de tipo bimodal para las zonas al norte y al sur del 
Tipología y usos del suelo en agroecosistemas del valle del guamuez, Putumayo - Colombia

río Guamuez de cerca de $3.600 \mathrm{~mm}$ anuales, con ocurrencia de dos intervalos menos lluviosos entre enero-febrero y junio-julio. El promedio anual de temperatura es de $28^{\circ} \mathrm{C}$ que permanece casi constante a través del año. La variación de altitud es de 250 a $290 \mathrm{msnm}$. La región dispone solamente de 4.5 horas diarias de radiación solar directa, valor límite para cultivos de cacao, cítricos y piña.

Para la definición de los sistemas de producción del área visitada se priorizó el suelo como la base fundamental de las relaciones culturales y sociales de la población rural en el territorio. En la zona se distribuyen dos tipos de suelos: por un lado, en el paisaje de lomerío aparecen suelos predominantemente de tipo oxisol - ver Figura 2 (a) - , caracterizados por un juego simple de horizontes
Ap / Bw1 / Bw2, bajos a muy bajos contenidos de materia orgánica, incluso en el horizonte Ap, ácidos, desaturados, de coloraciones pardo a pardo amarillento en superficie y pardo fuerte a pardo rojizo en profundidad y fertilidad general baja. En zonas depresionales los suelos son mal drenados, con evidencias de gleyzación.

En las terrazas (bajas, medias y altas), se encuentran suelos de tipo inceptisol - ver Figura 2 (b) -, con horizontes Ap / Bw de mayor grosor, en que se evidencian contenidos moderados a altos de materia orgánica y coloraciones más oscuras (pardos grisáceos oscuros ensuperficie a pardo fuerte en profundidad). En general estos suelos son de fertilidad moderada.

FIGURA 2: Perfil de suelos Oxisoles (a) e Inceptisoles (b) donde se desarrollan los sistemas de producción de lomerío en Puerto Asís y en el Valle del Guamuez

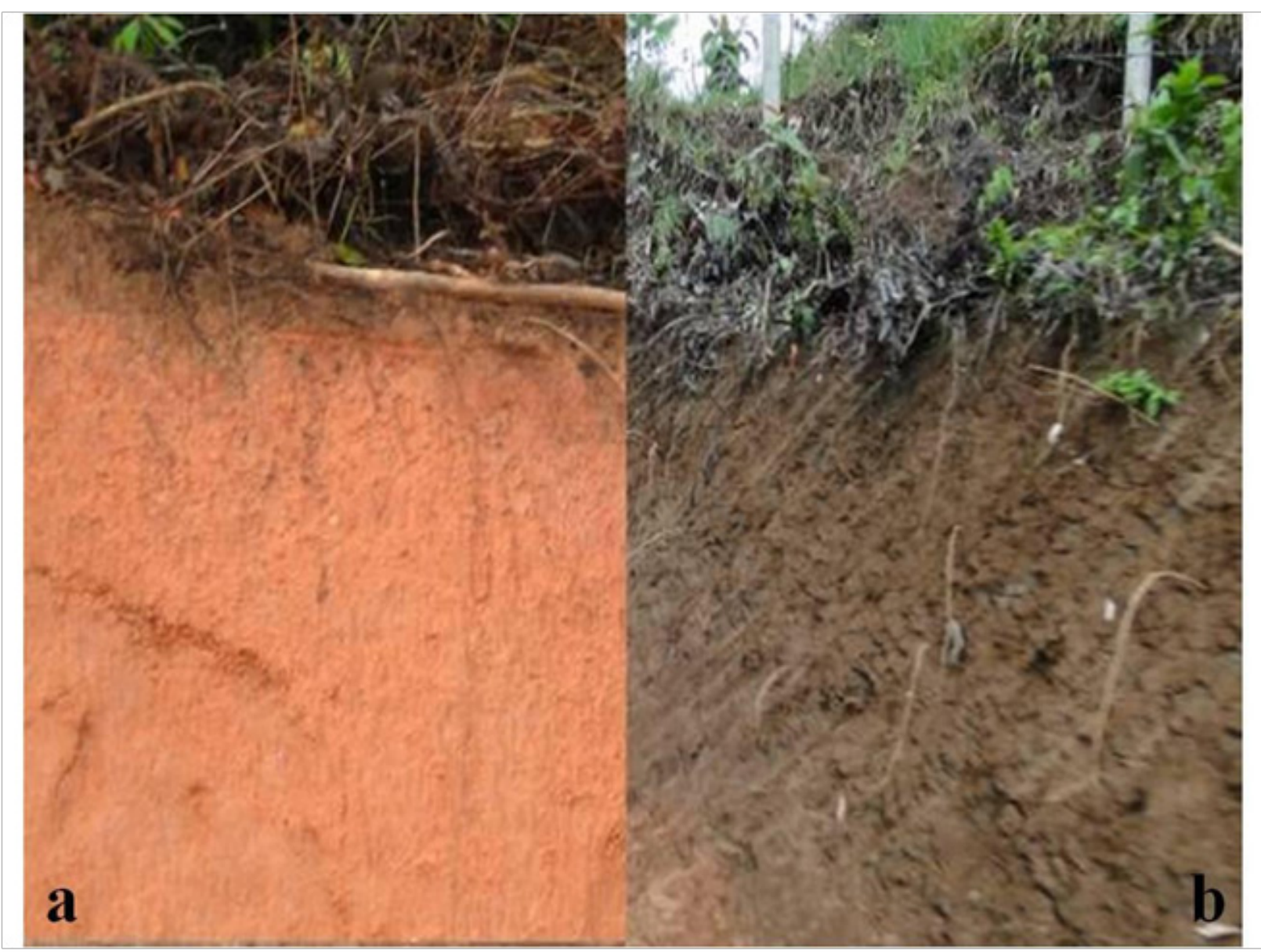

Fonte: Os autores (2014) 
En esta zona se ubica una población de campesinos procedentes de varios departamentos del país, compuesta por mezcla de mestizos, comunidades indígenas $\mathrm{y}$ organizaciones comunitarias de afrodescendientes. La actividad agropecuaria se viene desarrollando principalmente en las zonas de Valle del Rio Putumayo y en el Valle del Guamuez, entre otros.

Otras actividades económicas importantes en la región son: 1) la agroindustria del palmito para exportación aproximadamente en 700 has y 2) explotación de petróleo, enviado a través de un oleoducto hasta el municipio de Tumaco y de allí es llevado fuera del país vía marítima.

\section{Los sistemas productivos de la región estudiada}

En la zona coexisten sistemas de producción de pasturas, cultivos y diseños agrosilvopastoril, en diferentes paisajes fisiográficos y manejados por distintos grupos humanos. Entre los sistemas más representativos de la zona están:

\section{Sistema de pasturas en lomeríos, con suelos oxisoles, manejados por población mestiza}

Hacia el norte del Río Guamuez se encuentran suelos de lomerío en terrenos ondulados, con una capa arable (horizonte A) delgada de 10 centímetros aproximadamente, seguida por un horizonte subsuperficial $\mathrm{AB}$, de textura arcillosa (caolinita) y sin estructura, con muy bajo desarrollo radicular. Es manifiesta su alta susceptibilidad a la erosión. Hay presencia de remociones masivas pequeñas y permanentes, así como señales de poca capacidad portante con efecto de daño erosivo conocido como "pata de vaca" (ver Figura 3). La vegetación predominante son pasturas sin presencia de árboles y dedicadas a la explotación ganadera extensiva. No se observa desarrollo de otros cultivos y no se dispone de estadísticas sobre tamaño promedio de los predios ni gastos en insumos y costo de mano de obra. La tecnología de manejo de pastos se reduce a mantener un control de arvenses y establecimiento de cercas.

La población establecida en este tipo de sistemas productivos son colonos en su mayoría, procedentes de otras regiones del país, que por presiones de violencia y situaciones de inseguridad en las regiones se desplazan para buscar nuevas oportunidades.

FIGURA 3: Relieve de lomerío en suelos oxisoles en Puerto Asís, Putumayo

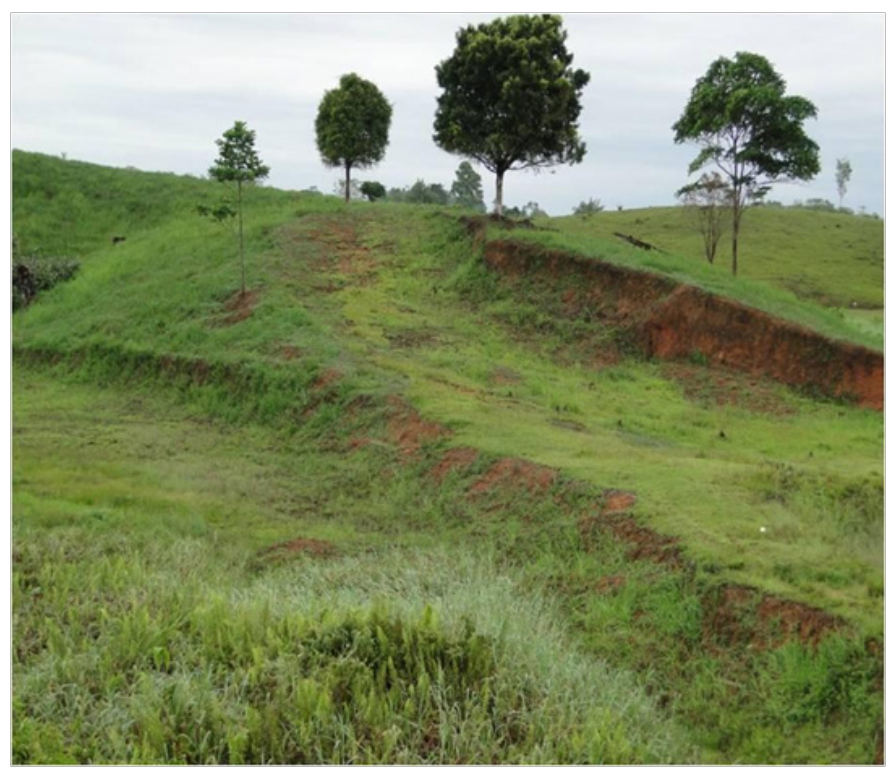

Fonte: Os autores (2014). 
2. Sistemas de producción de cultivo en terrazas y valles de los ríos Putumayo, Guamuez y Orito de población mestiza

Este sistema se desarrolla en suelos inceptisoles y entisoles, de moderado contenido de materia orgánica y una profundidad efectiva promedio de $100 \mathrm{cms}$. La aptitud productiva desarrollada está representada en cultivos de cacao-plátano, maíz, palmitos, chontaduro, silvopastoreo, yuca, cítricos, y otros árboles frutales.

En los últimos años, varios productores de las zonas de lomerío, como en el caso del municipio de Puerto Asís iniciaron procesos de conversión en sus fincas de potreros de solo pasto, hacia sistemas diversificados en arreglos silvopastoriles (Figura 4). Esta iniciativa fue promovida por CORPOAMAZONIA, el Comité de Ganaderos y la WWF (World Wildlife Fund). A este trabajo se le ha denominado "ganadería sin ganado", es decir, pensar en la explotación ganadera pero no antes de incorporar practicas de recuperación de suelos degradados por la potrerización y buscando un aumento de la biodiversidad funcional y alimentaria para los animales.

Los arreglos silvopastoriles en estas zonas consisten en el asocio de árboles forrajeros y maderables como matarratón (Gliricidia sepium) y nacedero (Trichanthera gigantea) con pasto braquiaria (Brachiaria decumbens) (ver Figura 4). Los resultados a la fecha han sido altamente significativos y se evidencia la recuperación los suelos de estas fincas permitiendo así el vigor y la densidad del pasto y en general de los alimentos para los animales, que antes de estas actividades solo dejaba fincas abandonadas por la deficiencia de forraje y alimento en general para el ganado.

FIGURA 4: Sistema agrosilvopastoril para ganados y cultivos en vegas y terrazas

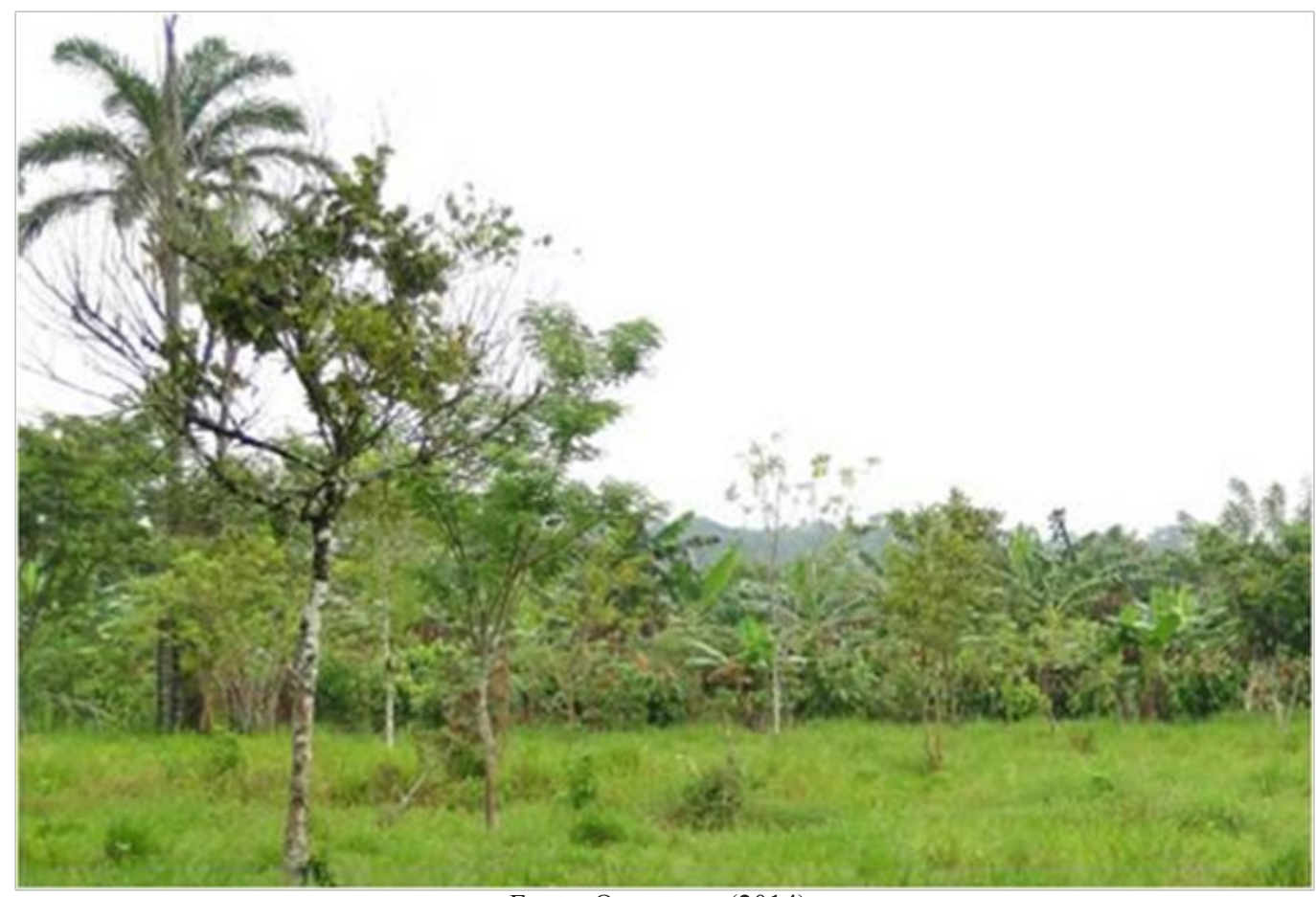

Fonte: Os autores (2014)

3. Sistema de producción agrosilvopastoril en lomas $y$ vallecitos manejados por culturas indígenas y afro descendientes

Este sistema se desarrolla en suelos inceptisoles de lomas y vallecitos (superficies cóncavas entre lomas), bien drenados, de buena profundidad efectiva, moderado contenido de materia orgánica con una explotación de bosque y alta diversidad productiva. Es un sistema con características altamente diversas 
Tipología y usos del suelo en agroecosistemas del valle del guamuez, Putumayo - Colombia

Juan Antonio Espinosa-Alzate, Tomás Enrique León-Sicard, Leonardo Alberto Ríos-Osorio

que imitan los ecosistemas naturales, de subsistencia y sin uso de insumos químicos o tecnologías que afecten el ecosistema. (ver Figura 5)

Los sistemas de producción identificados a nivel de microrregión indican un amplio contraste de opciones productivas. En el sistema de pastos y ganados bajo oxisoles (ver Figura 3), existe una gran limitación por la alta susceptibilidad del suelo a la erosión, baja fertilidad y la baja capacidad productiva. Estos resultados se ven reflejados en el escaso desarrollo de los pastos y los claros y frecuentes deterioros por erosión. En este tipo de erosión es determinante el material parental. Cualquier intervención debe considerar esta condición para definir el tipo de manejo y de especies a incorporar.

El sistema de producción de pastos y diversos cultivos en terrazas y valles ofrece un amplio margen de aptitud para el desarrollo de los cultivos (ver Figura 5), pues las condiciones de topografía y suelos proporcionan diversidad y fácil manejo de la producción. La diversidad de especies, el desarrollo de los cultivos y la vegetación indican la capacidad y potencialidad productiva de estos suelos. Un factor negativo es el mayor riesgo a daños por inundaciones y a ser fumigados con glifosato para control de coca, que las autoridades en la región realizan sin medir consecuencia alguna.

FIGURA 5. Sistemas productivos altamente biodiversos de culturas afro descendientes en Puerto Asís

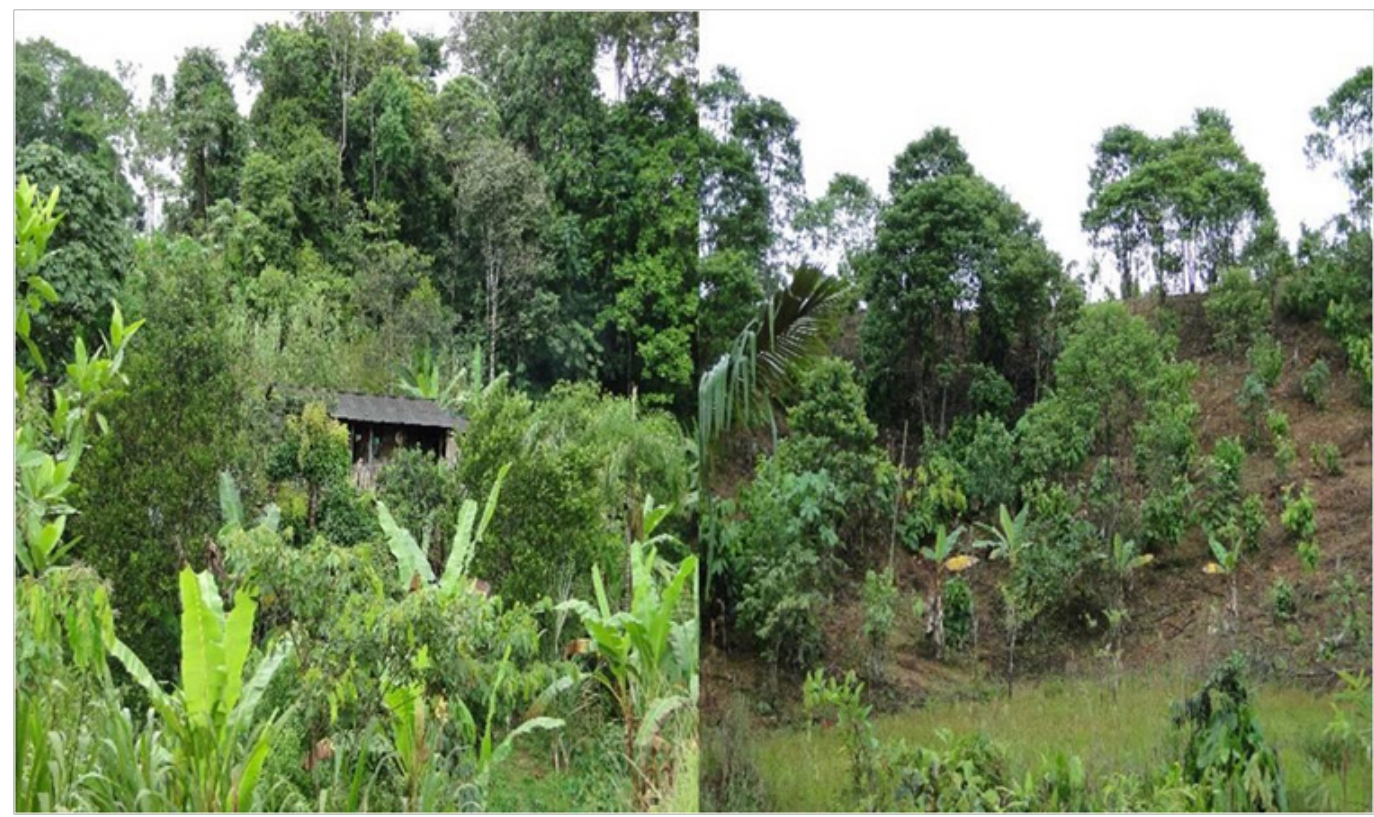

Fonte: Os autores (2014).

\section{DISCUSIÓN}

Usos culturales de la tierra: aportes para la comprensión de los sistemas productivos y los procesos de deterioro del suelo

En la región de estudio coexisten grupos de indígenas con colonos y población flotante que poseen modos de vida completamente diferentes. Los indígenas giran culturalmente alrededor de sus tradiciones milenarias, conocen los ciclos y ritmos del ecosistema, poseen instrumentos culturales poderosos para vivir de manera más que autosuficiente con los recursos que les provee el medio y sus aspiraciones excluyen, por lo general, la acumulación de riquezas, aunque ello no significa que renuncien a insertarse en los procesos de bienestar que les puede ofrece la sociedad occidental. Toledo (1980), resalta que durante la modernización, los recursos naturales y las comunidades campesinas tienden a ser destruidos y reemplazados por formas "modernas" de producción, basadas en una especialización espacial, productiva 
y humana y en una producción exclusivamente orientada al mercado, donde las relaciones hombre - naturaleza pierden su dimensión afectiva y donde están implicados grandes costos ecológicos.

Los campesinos y colonos, a su vez, pueden constituir un grupo heterogéneo de campesinos antiguos, asentados en la región desde hace varias décadas y que han podido asimilar prácticas $\mathrm{y}$ costumbres indígenas (incluso por la vía del parentesco mediada por matrimonio o concubinato), mezclados con colonos nuevos, atraídos o no por el auge de los cultivos de coca. Este fenómeno, a su vez, genera atractivos para una masa de población flotante que depende de los ciclos de interdicción / bonanza de la coca y que se desplaza periódicamente en la región. Todos estos habitantes foráneos comparten aspiraciones de ingresos de capital que les permiten acumulación suficiente para distintos fines.

Esta heterogeneidad implica, por lo menos, dos modos de vida diferentes: uno, de tipo "endógeno" ligado a las comunidades indígenas que poseen aspiraciones de autonomía completa, soportadas en sus tradiciones milenarias, en la propiedad ancestral y comunal de la tierra, en el intercambio de productos y en la chagra indígena como centro de los usos del territorio complementada con la recolección, la pesca y la caza, pero que está seriamente amenazada desde hace por lo menos tres décadas, por las intervenciones de colonos, narcotraficantes y empresas petroleras. Estos tipos de vida "endógena", es una categoría de relaciones inter - étnicas que apunta hacia la construcción de modelos de vida alternativa y de sociedad. Implica un esfuerzo por explicar la diversidad biológica desde la perspectiva endógena de la lógica eco-cultural de la Amazonía colombiana (ESCOBAR, 2008).

El otro, mediado por la visión clásica del desarrollo económico, que empuja por igual los esfuerzos de los cultivadores y traficantes de coca y cocaína, de los campesinos asentados en las orillas de los ríos, y de distintas empresas productivas que han intentado crecer y prosperar en la región. Todos los habitantes, sin importar a qué modo de vida se inscriban, se ven abocados a superar las limitantes biofísicas y ecosistémicas del territorio, agravadas en la región por los procesos de tala y uso equivocado de las tierras, que han generado, en muchos sectores, procesos severos de degradación del medio natural incluyendo fenómenos irreversibles de erosión en surcos y cárcavas. Este deterioro se expresa en disminuciones significativas de recursos de pesca, caza y extracción de recursos forestales que limitan significativamente las posibilidades de subsistencia y de acumulación de los habitantes locales.

La política de erradicación forzosa de cultivos de uso ilícito de coca utilizando el herbicida glifosato, por parte del gobierno nacional, ha generado diversos efectos en los pobladores, algunos de los cuales han abandonado esta práctica y han emigrado y otros han optado por establecer cultivos de uso lícito. No obstante, algunos de estos esfuerzos se pierden por fumigaciones inconsultas que destruyen campos de arroz, cacao, plátano y otros cultivos que aportan a la seguridad alimentaria, colocando en riesgo tanto la credibilidad de los programas alternativos de desarrollo rural, como las mismas posibilidades físicas de subsistencia de comunidades vulnerables.

Finalmente, se encuentran asentados en la zona los afrodescendientes, población conformada por pequeñas comunidades que provienen de la zona de Tumaco y que llegaron a la región por el corredor geográfico que naturalmente une las dos regiones. Estas comunidades, aunque habitan la región del Putumayo poseen mayores relaciones sociales, culturales y comerciales con sus hermanos de la zona de Tumaco. Para ellos, los modos de vida asociados al uso del suelo están relacionados estrechamente con procesos extractivos y al igual que las comunidades indígenas, aunque carecen de la figura de la chagra como unidad primaria productiva, se acercan a procesos productivos similares de policultivos y de recolección. Los modelos locales de naturaleza de los grupos negros no están representados por la separación estricta entre los mundos biofísicos, humanos, y sobrenaturales; estos grupos no se ven a sí mismo manipulando la "naturaleza" por fuerza de la "cultura", como si lo hacen los modelos modernos (STRATHERN, 1980).

\section{La erradicación de la coca y su sentido territorial}

Los procesos forzosos de erradicación de coca, soportados en el Plan Colombia y apoyados 
Tipología y usos del suelo en agroecosistemas del valle del guamuez, Putumayo - Colombia

Juan Antonio Espinosa-Alzate, Tomás Enrique León-Sicard, Leonardo Alberto Ríos-Osorio

de manera directa por los recursos de la cooperación internacional, han traído consecuencias importantes para los pobladores colonos del Putumayo: En primer lugar, se ha venido dando de manera progresiva un cambio cultural en la concepción de la tierra y su tenencia, que pasó de ser el espacio único de producción de coca, al de un espacio múltiple para garantizar la supervivencia, en una nueva concepción de la tierra, de su cuidado y de su conservación, que ha llevado tanto a agricultores como ganaderos a pensar en sistemas como el agrosilvopastoril y los sistemas productivos de base agroecológica como alternativas de mayor viabilidad. Esta situación responde igualmente a una serie de procesos ecosistémicos y culturales complejos, como la ausencia del Estado, el aislamiento geográfico, la lejanía de los centros de poder y de comercio y las particularidades climáticas, edáficas y geomorfológicas que, en su conjunto, hacen muy difícil la instalación y permanencia de usos alternos del suelo. León (2010), menciona que estas regiones, especialmente la del Putumayo (que pertenece a la gran cuenca Amazónica), solo se han podido vincular a los procesos de desarrollo económico a través de economías extractivas de quina, caucho, pieles, recursos mineros o animales, que por lo general causaron y causan efectos negativos en la existencia física de la selva o de sus habitantes nativos. La extracción de petróleo en la región de Orito - Puerto Asís ha causado violencia y muerte a las comunidades indígenas locales. La coca es un cultivo autóctono de las regiones amazónicas, utilizado durante milenios como vehículo transmisor de la cultura $\mathrm{y}$, por lo tanto, fuertemente arraigado a la cosmogonía y a las prácticas cotidianas de los pueblos amazónicos, entre ellos el putumayense.

En consecuencia, se ha generado un pensamiento ecologista y conservacionista y una conciencia de la autogestión, que ante la carencia de liderazgo de los gobiernos locales ha generado procesos de asociatividad que con el tiempo y el interés y liderazgo de algunos de sus representantes comunitarios se han logrado mantener y adaptar a las condiciones sociales, económicas y culturales de la región. Casos como el de ASOPA (Asociación de Cacaoteros de la Hormiga) y ASOPEZ (Asociación de Piscicultores del Valle del Guamuez), entre otros, han generado un nuevo panorama social y económico en la región, el cual requiere tanto de apoyo para fomentar su continuidad como su estudio por parte de grupos interdisciplinarios que permitan entender tanto su surgimiento como su consolidación para ayudar a definir estrategias de asociatividad adecuadas a las condiciones ecológicas, sociales y culturales de la región.

Finalmente, es importante resaltar como se percibe a la finca como unidad primaria productiva basada en un modelo de trabajo sustentado en la diversidad de sus sistemas de producción (granjas multidiversas), donde los productores tienen la posibilidad de concentrar su mano de obra en actividades que les generen abastecimiento de alimentación para sus familias y comunidades, garantizando la seguridad alimentaria, y diversas oportunidades económicas mediante la comercialización en mercados regionales, caso contrario a las iniciativas externas orientadas hacia la especialización por medio de monocultivos y que además son propuestas que en su mayoría no son consultadas o no tienen su origen en las comunidades.

\section{AGRADECIMIENTOS}

Este trabajo es producto de investigación financiado por el Programa MIDAS-USAID en el año 2010-2011.

\section{REFERENCIAS}

ÁNGEL, A. La trama de la vida. Bases ecológicas del pensamiento ambiental. Dirección General de Capacitación - Ministerio de Educación Nacional Colombia - Instituto de Estudios Ambientales (IDEA) - Universidad Nacional de Colombia, Bogotá, 1993. pp. 77.

ÁNGEL, A. La fragilidad ambiental de la cultura. Universidad Nacional de Colombia, Bogotá, 1995. pp. 127.

ÁNGEL, A. El reto de la vida. Ecosistema y cultura. Una introducción al estudio del medio ambiente. Ecofondo, Bogotá, 1996. pp. 109. 
ALTIERI, M. Agroecology: The science of sustainable agriculture. Westview, California University, Boulder, 1995.

ESCOBAR, A. Territorios de diferencia: lugar, movimientos, vida, redes. Duke University Press, Durham, 2008. pp. 153.

FAO. Land and Water Development Division. Planning for sustainable use of land resources. Towards a new approach. FAO Land and Water Bulletin 2. 1995.

GLIESSMAN, S. Agroecology: ecological Process in Sustainable Agriculture. Ann Arbor Press, Ann Arbor, MI. Lewis publishers, California, 1998.

LEÓN, T. Medio ambiente, tecnología y modelos de agricultura en Colombia: hombre y arcilla. Instituto de estudios ambientales, Universidad Nacional de Colombia, Eco ediciones, Bogotá, 2002. p. 166.

LEÓN, T. Análisis desde una perspectiva agroecológica, a la asistencia técnica en apoyo a los programas productivos agropecuarios con cofinanciación de USAID en Nariño y Putumayo. Universidad de Antioquia, Medellín. 2010.

STRATHERN, M. No Nature, No Culture: The Hagen Case. In: C. MACCORMACK, C., STRATHERN, M. (Eds.) Nature, Culture, and Gender. Cambridge University Press, Cambridge, 1980. p. 174-222.

TOLEDO, V. Ecología del modo campesino de producción. Antropología y Marxismo, 3, p. 35 - 55, 1980.

VAN DER HAMMEN, T., ANDRADE, G. Estructura Ecológica Principal de Colombia. Primera Aproximación. IDEAM. Bogotá. 2003. 